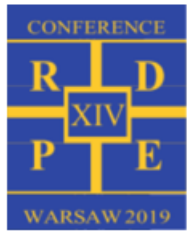

\title{
Experimental and numerical studies of sound generated by cavities
}

\author{
Sebastian Rulik ${ }^{1,}{ }^{*}$, Włodzimierz Wróblewski ${ }^{2}$, Mirosław Majkut ${ }^{3}$, Michał Strozik ${ }^{4}$, K rzysztof R usin ${ }^{5}$ \\ 1,2,3,4,5 Silesian University of Technology, Poland, sebastian.rulik@ polsl.pl,wlodzimierz.wroblewski@ polsl.pl, \\ miroslaw.majkut@polsl.pl, michal.strozik@ polsl.pl, krzysztof.rusin@ polsl.pl
}

\begin{abstract}
Cavities and gaps are an important element in the construction of many devices and machines, including energy sector applications. This type of flow is usually coupled with strong pressure fluctuations inside the cavity, which are emitted into the far field in the form of a sound wave responsible for the noise generation. This applies to both subsonic and supersonic flows. Pressure fluctuations often have the character of single tones of a specific frequency and high amplitude and their generation is associated with a vortex shedding formed directly above the inlet and its interaction with the walls of the cavity. The presented work include description of developed test stand and applied measurement techniques dedicated to the analysis of high frequency phenomena. In addition, the adopted numerical model will be described, including conducted two-dimensional and three-dimensional analysis. The developed models will be validated based on experimental measurements concerning wide range of flow conditions.
\end{abstract}

\section{Introduction}

A nalysis of the flow in the cavities is a subject of a numerous experimental and numerical investigations. This type of flow is usually accompanied by strong pressure fluctuations inside the chamber itself, which are emitted into a far field in the form of a sound wave responsible for the generation of noise. It concerns both subsonic and supersonic flows. Pressure fluctuations are often characterized by a single mod with a certain frequency and high amplitude. Their generation is associated with the vortex path created directly above the cavity inlet and its interaction with cavity walls.

This type of flows occurs in many machines and devices. The most popular examples include: cavities in a landing gear of plane, bomb hatches, open car windows, gaps in car bodies. These examples pertain open flows. Besides, this type of flow occurs in many parts of a gas or steam turbine blade system such as labyrinth seals or rim seals applied between stator and rotor $[4,5]$ and spaces between guiding vanes and turbine casing resulting from its modular construction [12]. A nother example is flame stabilizer applied in the ramjet engines. In all aforementioned cases, there are closed flows in which the influence of the flow channel walls on the mechanism of generation and propagation of disturbances should be additionally considered.

References $[9,10,11]$ supplement research of this type of phenomenon with the relation between the parameters of the generated acoustic wave and the conditions of heat exchange inside the chamber. The paper presented herein is a part of the validation process of the applied numerical models based on conducted experimental investigations.

\section{Investigated phenomenon}

Mechanism of tonal noise generation inside the cavity, which is flown over by gas was described by Rossitier in the sixties of the past century [7]. It is based on the feedback loop between generated acoustic wave and the flow of subsequent vortices from the leading edge of the chamber.

Nowadays, this type of flow is a frequent subject of investigations. The main motivation is understanding of the phenomena responsible for the generation of the strong pressure pulsations, which subsequently will make it possible to reduce noise and may contribute to the improvement of unfavourable working conditions of machines, in which this phenomenon occurs.

Rossiter [8] was first to discover that the frequency of pulsation was connected with feedback loop mechanism. His observations with the use of shadowgraph technique showed the presence of large vortex structures responsible for pressure pulsations inside the cavity.

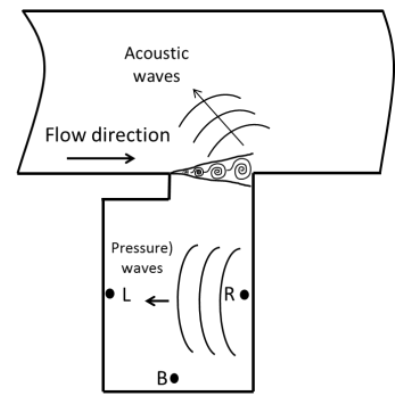

Fig. 1 Feedback loop mechanism

Their interaction with the rear cavity wall causes generation of pressure pulses which, moving towards the front wall of the cavity, intensify the flow of subsequent 
vortices. Part of the generated waves escapes the cavity and is audible as noise. This mechanism is presented for a cavity with additional cover in Fig. 1. Rossitier introduced also half-empirical formula which makes it possible to determine frequency of generated tonal noise:

$$
S t=\frac{f L}{U_{\infty}}=\frac{m-\alpha}{M_{\infty}+\frac{1}{k}} \quad m=1,2,3 \ldots
$$

Quantity $k$ is a convective velocity of the vortices related to the main flow velocity and $\alpha$ is a delay time between the vortex impingement onto the cavity wall and moment of acoustic wave generation. In most cases $k$ is within range $0.5-0.75$ and $a \quad 0-0.25$ [13]. Both quantities are chosen empirically. It is worth noting that in the presented equation Strouhal number, that is the dimensionless frequency of pressure pulsations for a given mod, is a function of only $M$ ach number and two constant $a$ and $k$.

A part from the mechanism of sound wave generation based on resonance associated with feedback loop between frequency of generated vortices and frequency of acoustic waves, other types of resonance may occur, which significantly increase the level of generated sound. An example of such a resonance, which may occur in discussed flow, is a cavity working as Helmholtz resonator [2]. In this case air column inside the cavity is subjected to periodic compression and expansion which is associated with the compressibility of air. The resonating frequency of generated acoustic wave should be independent of free stream velocity [1]. This type of resonance occurs with relatively low $\mathrm{M}$ ach numbers $(M<0.2)$, particularly for deep cavities (Length/Depth<2) and the ones with covered inlet. Frequency of acoustic wave generated by the Helmholtz resonator can be expressed as:

$$
f=\frac{a}{2 \pi} \sqrt{\frac{C}{V b_{e f}} .}
$$

The frequency of Helmholtz resonator can be also determined from equation (3) which is a modified version of the equation proposed by Rayleigh $[5,6]$.

$$
f=\frac{a}{2 \pi} \sqrt{\frac{C}{V\left(b+\frac{1}{2} \sqrt{S \pi}\right)} .}
$$

In equations 2 and $3 a$ is speed of sound, $C$ is area of inlet cross section, $V$ is cavity volume, $b$ is height of cavity neck and $b_{\text {ef }}$ is effective height of the cavity neck which depends on $b$ and cavity geometry.

For cavities with a partially covered inlet, pressure pulsations resulting from the feedback mechanism and pulsations associated with the operation of the cavity as a Helmholtz resonator can occur simultaneously, leading to its intensification [3].

\section{Test stand}

Presented experimental investigations were performer in the Fluid Flow Machines Laboratory of
Institute of Power Engineering and Turbomachinery. The laboratory is equipped with the air system operating in underpressure. The diagram of air system is presented in Fig. 2. The main part of the installation is Roots blower DR124T with maximum volumetric flow rate equal to $12.4 \mathrm{~m}^{3} / \mathrm{min}$. It is propelled by $13.2 \mathrm{~kW}$ synchronous electric engine and maximum under pressure obtained is $50 \mathrm{kPa}$. The Roots blower is directly connected with the $3.2 \mathrm{~m}^{3}$ air tank. This tank is designed to level pressure pulsations associated with the operation of the blower. Moreover, the blower is placed inside of the soundshield. The other parts of air installation consist of aluminum piping with $105 \mathrm{~mm}$ in diameter.

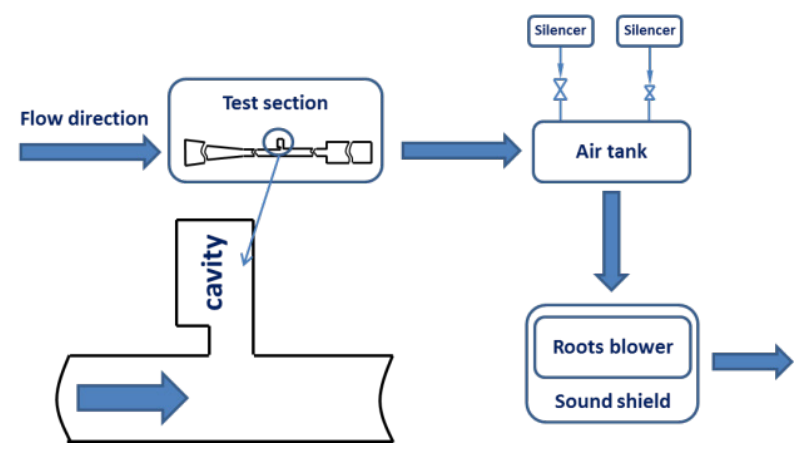

Fig. 2 A ir system

The test rig constitutes a first element of the presented air installation operating in underpressure. It consists of 3 main parts (Fig. 3). The first one is converging inlet with an opening angle equal to $6^{0}$. The next part is a rectangular channel with the dimensions of cross-section equal to $50 \times 40 \mathrm{~mm}$, in which the investigated cavity is installed in the middle. The final part of the installation is the transition from a rectangular cross-section to a circular one and the connection with air supplied system.

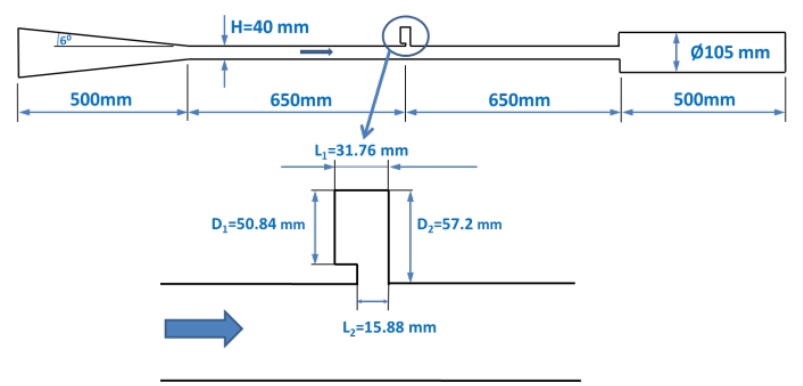

Fig. 3 Diagram of test rig and its dimensions

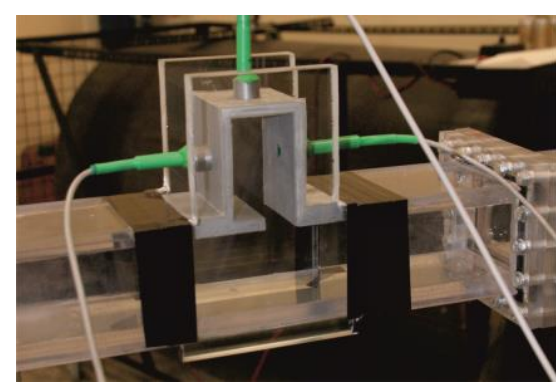

Fig. 4 Investigated cavity

The test stand is made out of polycarbonate and sidewalls of the cavity are demountable. The deep cavity 
with partially covered inlet is placed in the upper part of the main channel (Fig. 4).

A set of $6 \mathrm{~mm}$ microphones was prepared for measuring acoustic signals. Each of the microphones works with a separate microphone amplifier with adjustment. The calibration of the microphone measurement track was carried out on a specially prepared stand with a high-power pistonphone. The set of eight microphone amplifiers works with the $\mathrm{N}$ ational Instruments NI USB-6216 measuring module. It is a universal system containing a 16-channel, 16-bit AC converter with $400 \mathrm{kS} / \mathrm{s}$ sampling and communication with a master computer via a USB serial port. The mass flow rate measurement was carried out using an orifice and a calibrated thermoanemometer. In addition, a Pitot tube was installed in the flow channel to measure velocity in the channel axis.

\section{Numerical model}

Numerical analyses were carried out using various models differing in size and geometry of the analyzed computational area. The analyses were performed on both simplified two-dimensional models and threedimensional models. The basic configuration of the computational area for two-dimensional analyses is presented in Fig. 5. The chamber in this case is located in the middle of the $40 \mathrm{D}_{1}$ main channel.

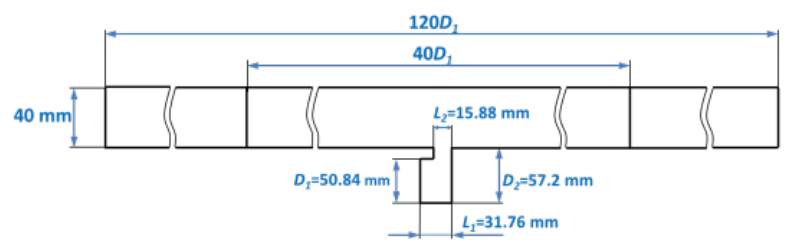

Fig. 5 Computational domain

Two-dimensional calculations were carried out based on two models (Fig. 6), aimed at eliminating the reflection of the acoustic wave from the edge of the computational domain. One method is to use non-reflecting boundary conditions (model 1, Fig. 6). This concept for high-amplitude waves is often unreliable, and its application can lead to instability of the calculation process, hence it was proposed to replace non-reflective boundary conditions with an additional buffer domain at the inlet and outlet with a relatively rare numerical mesh. Acoustic waves after passing through this area are further attenuated due to numerical dissipation, which prevents strong reflections from the inlet and outlet areas of the main channel. Such a solution requires, however, both an increase in the number of computational nodes and an extension of the entire computational area to the length of $120 D_{1}$ (Fig. 6). The additional domain has been connected to the main channel with the use of General Grid Interface. In the buffer domain for the bottom and upper surface, the boundary condition of the free slip wall has been adopted, hence this area does not cause additional losses in the flow channel that result from fluid viscosity. B oth analysed variants of the computational domain were compared and the obtained results are very similar. The second solution, however, is more stable for all considered flow conditions and configuration of boundary conditions. Due to the inability to conduct purely two-dimensional analyses, the calculation area for models 1 and 2 has a span of $1 \mathrm{~mm}$. The boundary conditions adopted for analysis are presented in Table 1.
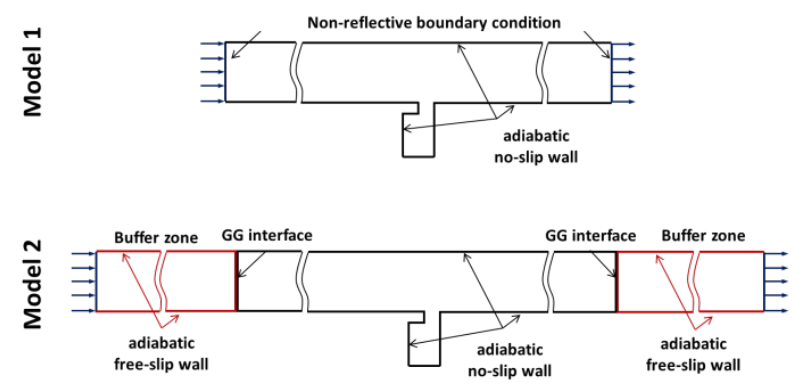

Fig. 6 Boundary conditions adopted to CFD analyses

The boundary condition of symmetry was assumed for the lateral walls of the channel and no-slip wall was adopted for the upper and bottom surfaces.

The numerical mesh is shown in Fig. 7. It is a structural, orthogonal mesh with 231,000 nodes. The span of the flow channel is discretized with a single element. The mesh is significantly finer at the cavity inlet. This area is particularly important for correct modelling of generation and propagation of acoustic waves. For all walls of both the main channel and the cavity, the dimensionless val ue of $\mathrm{y}_{+} \approx 1$.

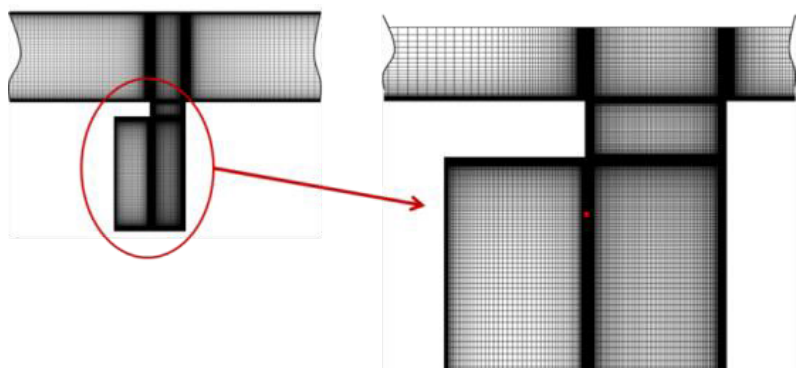

Fig. 7 Numerical mesh for CFD analyses

Tab 1 B asic boundary conditions and assumptions of the numerical analyses

\begin{tabular}{|l|l|}
\hline Inlet & $\begin{array}{l}\text { V elocity: } 50 \mathrm{~m} / \mathrm{s} \\
\text { Static temperature: } 15^{\circ} \mathrm{C} \\
\text { Turbulence intensity: } 1 \%\end{array}$ \\
\hline Outlet & A veraged static pressure: 1 bar \\
\hline $\begin{array}{l}\text { Channel and } \\
\text { cavity walls }\end{array}$ & A diabatic \\
\hline Lateral walls & Symmetry \\
\hline Fluid & Air ideal gas \\
\hline Heat transfer & $\begin{array}{l}\text { Energy equation based on total enthalpy with } \\
\text { taking account viscous work associated with } \\
\text { tangential stresses }\end{array}$ \\
\hline $\begin{array}{l}\text { Turbulence } \\
\text { model }\end{array}$ & SST or DES \\
\hline
\end{tabular}

The numerical mesh is gradually coarsened in the direction of inlet and outlet from the main channel. For 
model 2 (Fig. 6) in the interface area, the element size of the numerical mesh is abruptly changed. The mesh in the buffer domain is not refined along the channel edge due to the accepted boundary condition of free slip wall.

The third investigated numerical model (model 3) relates to calculations using methods based on filtered Navier-Stokes equations based on the DES method. It is assumed that the configuration of the computational domain and boundary conditions correspond to model 1 (Fig. 6), while the span of the computational area is extended to $20 \mathrm{~mm}$, which allow for the threedimensional character of the flow. In this case, the span of the flow channel and the cavity is discretized using 50 elements of the numerical mesh, which together gives a grid of 5.8 million nodes.

Models $4 a$ and $4 b$ relate to three-dimensional calculations using the averaged $\mathrm{N}$ avier-Stokes equations. The model k- $\omega$ SST was used to model the Reynolds stress tensor. In this case, the geometry of the computational domain exactly matches the dimensions of the test stand (Fig. 3). In the 4a model, the free slip boundary condition was adopted for the lateral walls of the computational domain, which allowed limiting the size of the numerical mesh. For model $4 \mathrm{~b}$, the numerical mesh was refined near the lateral walls of the domain and the no-slip boundary condition was assumed. This made it possible to correctly determine the velocity distribution along the span of the flow channel.

\section{Results}

Computations were carried out for a flow velocity in the channel equal to $50 \mathrm{~m} / \mathrm{s}$. For the simulation of non-stationary flow, a time step of $10^{-5} \mathrm{~s}$ was adopted, which gave 87 time steps for one acoustic wave period. The exception is time discretization used for the DES method. In this case, a time step of $2.5 \cdot 10^{-6} \mathrm{~s}$ was adopted, which provided 348 time steps for one period of pressure pulsation in the cavity. The process of determining the amplitude of the generated acoustic wave required approximately 14,000 iterations, which considerably increases computational time.

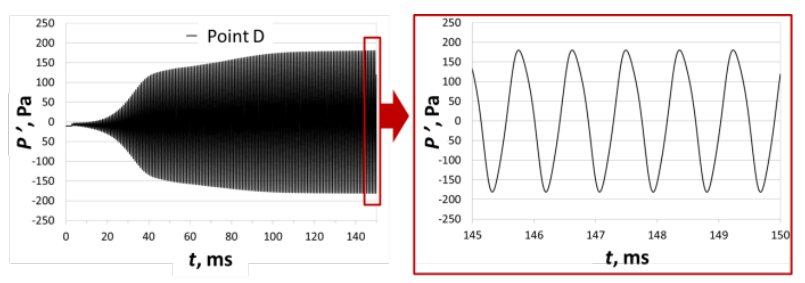

Fig. 8 Generation of sound pressure fluctuation at a $50 \mathrm{~m} / \mathrm{s}$ flow velocity - M odel 1

Fig. 8 shows the process of determination of acoustic wave amplitude. The obtained results concern two-dimensional analysis. Fig. 9 and 10 show the course of changes in pressure fluctuations for three control points located in the middle of the left, right and bottom walls (Fig. 1). These are respectively points $L, R, D$

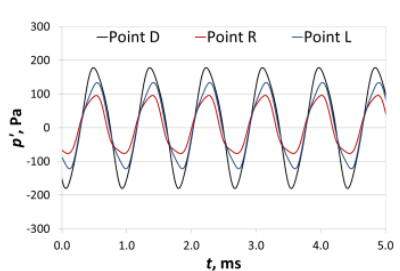

a) 2D k- $\omega \mathrm{SST}$ (model 1)

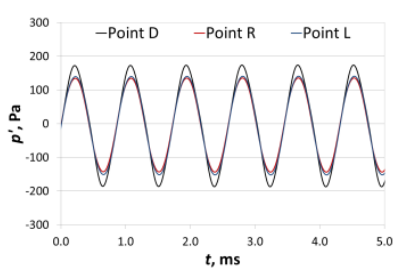

b) 3D k- $\omega \mathrm{SST}$ (model 4b)
Fig. 9 A coustic pressure fluctuations for flow velocity in the channel equal to $50 \mathrm{~m} / \mathrm{s}-\mathrm{k}-\omega \mathrm{SST}$

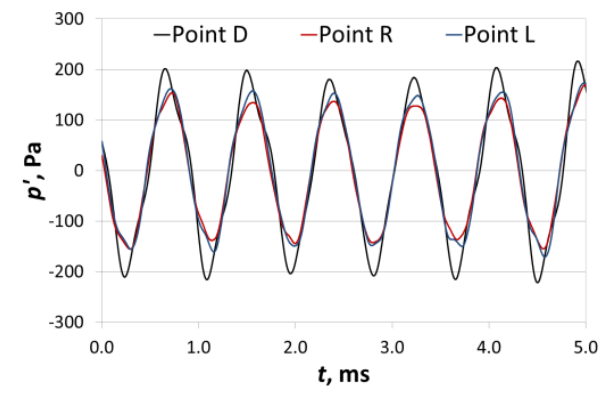

Fig. 10 A coustic pressure fluctuations for flow velocity in the channel equal to $50 \mathrm{~m} / \mathrm{s}-$ DES

The results of two and three-dimensional analysis based on the k- $\omega$ SST turbulence model and on the DES method are compared. In addition, the corresponding results of experimental studies are presented in Fig. 11. A fragment of the pressure pulsation course in the case of experiment covers a wider period of time, equal to $20 \mathrm{~ms}$. In this case, the course of fast-changing signals was recorded at a frequency of $44 \mathrm{kHz}$. It can be seen in Fig. 11 that the sound pressure oscillations at $50 \mathrm{~m} / \mathrm{s}$ are strongly periodical. The pressure course for all three analysed measuring points are sinusoidal. The analysis of measurement data also shows that the amplitude of acoustic pulsation for individual points remains almost unchanged. The obtained sound pressure fluctuation courses for all analysed numerical models are very similar and all are also characterized by ideal periodicity of the analysed phenomenon, which is also reflected, as mentioned earlier, in the obtained experimental data.

It is also important to note that for all three measuring points, the course of sound pressure fluctuations are coincidental in phase in spite of the slight differences in the pulsation amplitude. This indicates that this type of cavity acts as a Helmholtz resonator, and pressure pulsations occur uniformly throughout the cavity volume. However, this does not exclude the existence of an additional type of resonance resulting from the feedback mechanism described by Rossiter [8].

It is also worth noting that the results obtained from 2D simplified model and 3D model are in good agreement. The highest sound pressure level was obtained for point $D$, located on the bottom wall of the cavity, while the lowest for point $R$, located on the right wall. The relatively low sound pressure level for this point may be due to the fact that it is located much below the place where the vortices formed in the cavity inlet hit, hence the higher pressure pulsations on the right wall of the cavity will be observed rather in the vicinity of the 
main flow channel. The biggest difference between the sound pressure levels for the analyzed measuring points was obtained in the case of $2 \mathrm{D}$ analysis. The sound pressure fluctuation course for the DES are slightly sharper than in the case of other analyzed numerical models. It is also worth mentioning that the sound pressure fluctuations for the $4 \mathrm{a}$ and $4 \mathrm{~b}$ models are very similar, hence only one of the results obtained is presented.

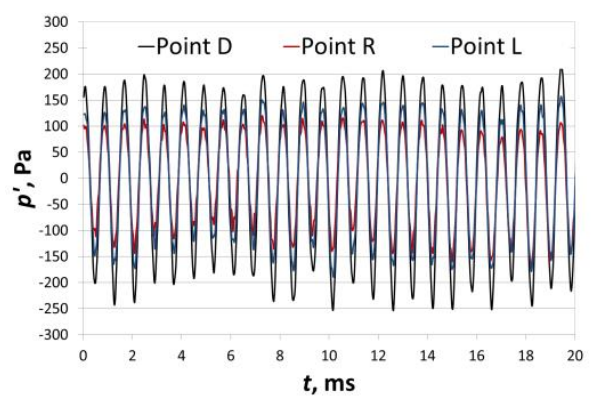

Fig. 11 A coustic pressure fluctuations for flow velocity $50 \mathrm{~m} / \mathrm{s}$ - experimental investigations

Non-stationary changes in sound pressure fluctuations obtained in experimental studies and from numerical calculations were subjected to FFT (Fast Fourier Transformation) spectral analysis. The sound pressure level spectra determined for each case calculated on the basis of numerical analyses are presented in Fig. 12, while in Fig. 13 the spectrum obtained on the basis of experiment is shown.

4096 samples after the stabilization of the acoustic pulsation level were taken into account for the FFT analysis. The FFT analysis of the sound pressure level in the case of experimental studies was additionally averaged. 10 separately performed spectral analyses of one continuous time period were taken into account, which were then averaged. Due to the relatively small changes in the level of pressure pulsation in time, the average FFT results do not differ significantly from the instantaneous course. A veraging, however, allows the elimination of individual sound pressure pulsations that deviate significantly from the mean values.

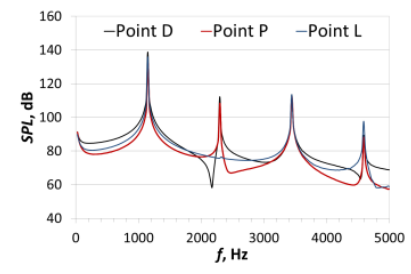

a) $2 \mathrm{D} \mathrm{k}-\omega \mathrm{SST}$ (model 1)

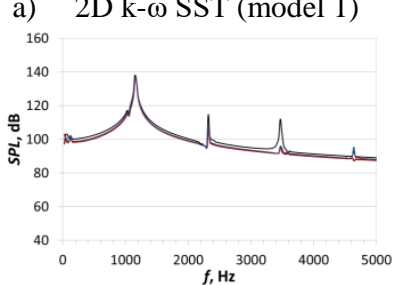

c) 3D k- $\omega \mathrm{SST}$ (model 4a)

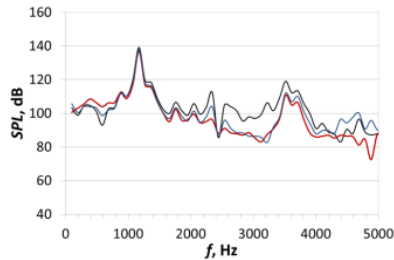

b) 3D DES (model 3)

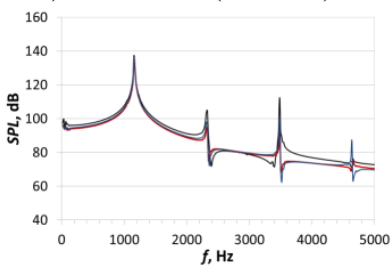

d) 3D k- $\omega \mathrm{SST}$ (model 4b)

Fig. 12 Spectral analysis of the sound pressure level for fluid velocity $50 \mathrm{~m} / \mathrm{s}$ - numerical investigations

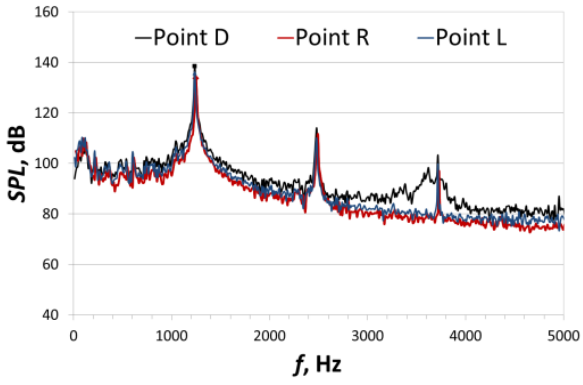

Fig. 13 Spectral analysis of the sound pressure level for flow velocity $50 \mathrm{~m} / \mathrm{s}$ - experimental investigations

All applied models based on averaged $\mathrm{N}$ avier-Stokes equations give a very smooth picture of the sound pressure level spectrum, while in the case of the DES method, the spectral characteristics include many smaller peaks associated with the modelling of smaller turbulence scales. In this case, it was also possible to capture small differences in the course of spectral characteristics near the third harmonic, which are also visible in experimental studies (Fig. 13). Nevertheless, the correct prediction of the amplitude and the frequency of the first mod is crucial due to the almost sinusoidal nature of the generated acoustic wave. A detailed comparison of the parameters of the first three mods is presented in Table 2.

Tab. 2 Frequency and acoustic pressure level for selected mods

\begin{tabular}{|c|c|c|c|c|c|}
\hline \multicolumn{7}{|c|}{$f, \mathbf{H z}$} \\
\hline Mod & Experiment & $\begin{array}{c}\text { DES } \\
\text { model } \\
3\end{array}$ & $\begin{array}{c}\text { k- } \omega \text { SST } \\
\text { model 4a } \\
3 D\end{array}$ & $\begin{array}{c}\text { k- } \omega \text { SST } \\
\text { model 4b } \\
3 D\end{array}$ & $\begin{array}{c}\text { k- } \omega \text { SST } \\
\text { model 1 } \\
2 D\end{array}$ \\
\hline 1 & 1235 & 1171 & 1147 & 1156 & 1148 \\
\hline 2 & 2492 & 2344 & 2319 & 2311 & 2295 \\
\hline 3 & 3717 & 3516 & 3467 & 3483 & 3442 \\
\hline
\end{tabular}

\begin{tabular}{|c|c|c|c|c|c|}
\hline \multicolumn{7}{|c|}{ SPL, dB } \\
\hline M od. & Experiment & $\begin{array}{c}\text { DES } \\
\text { model 3 } \\
\text { 3D }\end{array}$ & $\begin{array}{c}\text { k- } \omega \text { SST } \\
\text { model 4a } \\
\text { 3D }\end{array}$ & $\begin{array}{c}\text { k- } \omega \text { SST } \\
\text { model 4b } \\
\text { 3D }\end{array}$ & $\begin{array}{c}\text { k- } \omega \text { SST } \\
\text { model 1 } \\
\text { 2D }\end{array}$ \\
\hline 1 & 138.5 & 137.7 & 137.9 & 137.4 & 138.8 \\
\hline 2 & 111.4 & 112.4 & 114.7 & 104 & 112.5 \\
\hline 3 & 103.3 & 119.0 & 112.0 & 111.9 & 113.6 \\
\hline
\end{tabular}

A nalysis of the data presented in Tab . 2 shows that all the numerical models under predict the frequency of mods. These differences for the first mod are in the range of $5.2-7.0 \%$ and are the smallest for the DES method, while for the third mod they are in the range of $5.4-7.4 \%$. N evertheless, the difference between obtained frequency and experimental data increases for subsequent mods. V ery good agreement was obtained between all numerical models and experimental investigations in case of sound pressure level. For the first mod the maximum differences are $1.1 \mathrm{~dB}$. The highest discrepancies were obtained for the third mod and they are equal to a maximum of $15.2 \%$, but the amplitude of the sound pressure level is small in comparison to the first mod. 


\section{Conclusions}

Performed investigations concerning analysis of the noise generation by the cavity showed good agreement between numerical models and experiment. In both cases the generated by the cavity acoustic wave is almost purely sinusoidal with one main mod.

The course of pressure fluctuations are coincidental in phase for three investigated measuring point located at the centre of each cavity wall. This indicates Helmholtz type resonance which can coincide with feedback mechanism described by Rossiter.

The comparison between CFD and experimental data shows that all investigated numerical models slightly under predicted frequency of the first mod of pressure fluctuations and the differences were within range 5-7\% depending on the model. The discrepancies in sound pressure level between numerical models and experiment were maximum $1 \mathrm{~dB}$.

Presented analyses made it possible to develop an optimal numerical model to shorten computational time. Further investigations will concern wider range fluid velocities and experimental investigations will also take into account heat transfer conditions in case of heated cavity. This will allow the validation of numerical studies on the heat transfer conditions in the cavities.

\section{Acknowledgements}

The presented research was conducted within the the Silesian University of Technology statutory research funds for young scientists.

\section{References}

1. De Jong, A.T., Bijl H., Investigation of higher spanwise Helmholtz resonance modes in slender covered cavities, The J ournal of the A coustical Society of A merica, vol. 128 (4), 2010, p. 1668-1678.

2. Durgin W., Graf H., Flow-excited acoustic resonance in a deep cavity: an analytical model, Proceedings of the Third International Symposium on Flow-Induced Vibrations and Noise, vol. 7, 1992, p. 81-91

3. Loh Ching Y., Computation of Low Speed Cavity Noise, NASA/CR-2004-212892, 2004.

4. Patinios M., Ong I.L., Scobie J.A., Lock G.D., Sangan C.M., Influence of Leakage Flows on Hot Gas Ingress, Journal of Engineering for Gas Turbines and Power, Vol. 141/ 021010-1, 2019

5. Patinios M., Scobie J.A., Sangan C.M., Owe J.M., Lock G.D., M easurements and M odeling of Ingress in a N ew 1.5-Stage Turbine Research Facility, J ournal of Engineering for Gas Turbines and Power, Vol. 139/ 012603-1, 2017

6. Rayleigh L., On the application of the principle of reciprocity, Proceedings of the Royal Society of London, vol. 25, 1877, p. 118-122.

7. Rayleigh L., The theory of sound, Dover Publications, $\mathrm{N}$ ew Y ork, revised edition, 1945.
8. Rossiter J.E., Wind Tunnel Experiments on the Flow over Rectangular Cavities at Subsonic and Transonic Speeds, Royal Aircraft Establishment, Technical Report 64037, 1964.

9. Rulik S., Wróblewski W., A Numerical study of the heat transfer intensification using high amplitude acoustic waves, A rchives of A coustics, vol. 43, 2018, p. 31-47

10. Rulik S., Wróblewski W., Nowak G., Szwedowicz., Heat transfer intensification using acoustic waves in a cavity, Energy, vol. 87, 2015, p. 21-30.

11. Rulik S., Wróblewski W., Rusin K., A numerical study of the relation between the acoustic generator geometry and the heat transfer conditions, A rchives of M echanical Engineering, vol. 65(1), 2018, p. 5-26.

12. Spura D., L ueckert J., Schoene S., Gampe U., Concept development for the experimental investigation of forced convection heat transfer in circumferential cavities with variable geometry, International J ournal of Thermal Sciences, vol. 96, 2014, p. 277-289.

13. Ünalmis Ö.H., Clemens N.T., Dolling D.S., Cavity Oscillation Mechanisms in High -Speed Flows, AIA A J ournal, vol. 42 (10), 2004. 